\title{
Emerging Aspects of Artificial Intelligence for Smart Life
}

\author{
A. Kavitha \\ Head \& Assistant Professor of Computer Science, Aditanar College of Arts and Science, Tiruchendur, Tamilnadu \\ Email id: kavithajeyavelmurugan2016@gmail.com
}

\begin{abstract}
Nowadays Artificial intelligence makes our life easy and comfortable that is hard to imagine that to survive our life without AI technology. We all know that Artificial Intelligence (AI) is a precious gift to human being. Recently it is used in robotics, education, agriculture, computer vision, cyber security, face recognition, speech recognition, self driving cars, medical image processing, biometrics, bioinformatics, satellite control, disease detection, drugs development, network developments, manufacturing, business, healthcare and medicine. In the digital era AI provides the best results in all most all the domains. This article helps to understand the emerging aspects of AI in various fields.
\end{abstract}

Keywords - Artificial Intelligence; Agriculture; Deep Learning; Medicine; Machine Learning; Natural Language Processing; Robotics.

\section{Introduction}

We need intelligence to solve a particular problem in efficient manner. Like human being machines having Artificial Intelligence (AI) can do thinking, learning, reasoning, makes meaningful decisions and solves the complex problem efficiently [1].

In the digital world smart machines are very much useful in our daily routine tasks; they become the needs of our life. Without a doubt, most things belongs to us are applications of AI such as smart phones, Air conditioners, Digital cameras, video games, traffic lights, refrigerators, self driving cars, drones, robots, autonomous vehicles and machines etc. These applications of AI are work on a "smart" technology. AI is a latest growing technology that makes our work very simple. We know that today's smart machines work millions of times faster than earlier machines.

Recently many researches are going based on Machine learning (ML) that is a subdivision of AI. It makes the systems to learn automatically and get better from their experience. ML is used to teach machines the way to handle the data more efficiently. We can see the machine learning algorithms are used in our daily routine applications. For example the popular search engine such as Google works on machine learning algorithm basis. In addition, machine learning algorithm is used in the social networking site for example most of the business people use Facebook for their products and services developments.

At present DL is the recent most growing research areas. DL algorithms are widely used in disease diagnosis, drugs development, machine translation, object identification, industrial automation, marketing research, medical research, social network filtering, image recognition and sentiment analysis and so on [2]. The following diagram Fig. 1 describes the subset of Artificial intelligence.

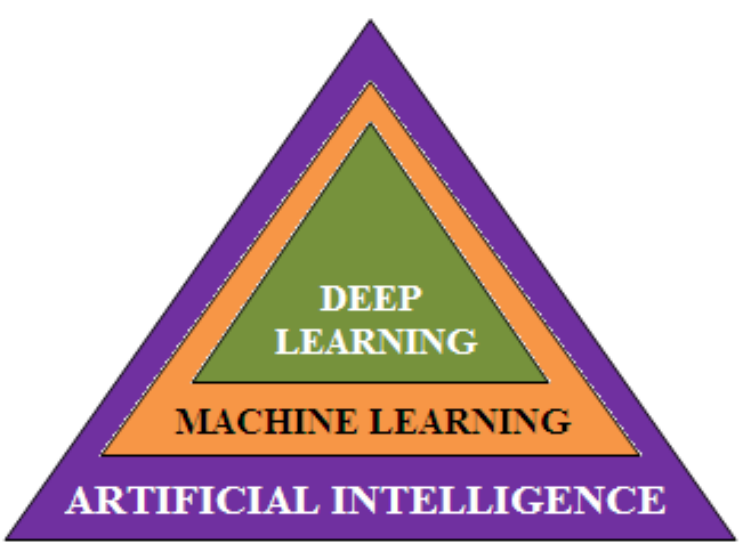

Fig. 1: Subset of Artificial Intelligence

\section{Advantages of AI}

- Handle large volume of data: Machine can handle large volumes of structured and unstructured data easily.

- Reduce the errors: Unlike humans smart machines do not make mistakes. Mostly it provides the accurate and error free result.

- Save our precious time: Smart machines can perform any types of work rapidly so it saves our precious time.

- Diligence: Unlike human being machine does not get tired and feel bored so it can work for 24 hours.

- Handle risky job easily: Smart machines can handle risky job very efficiently. For example robots are used in bomb deactivation, Space and ocean exploration.

- Take the decisions rapidly: Smart machine works with much higher speed compared to humans and produce the results in a faster way.

\section{Disadvantages of AI}

- Makes Humans Lazy: AI technology is making human being lazy because it automates the majority of the 
work. Nowadays humans are mostly relying on the smart machines that leads problem to future generations.

- Unemployment: AI technology replaces the robots for automation and risky job so it will create an unemployment problem. It affects the younger generation life.

- Lack of creativity: Machines can perform only the defined task that is written in the program by the user. It does not have creative thinking.

- Expensive: AI supporting machines required high expensive hardware and software. In addition we spend more money for maintenance [3].

\section{Applications of AI}

It is no doubt AI is used in numerous fields. Applications of Artificial intelligence are shown in Fig 2. Some of the widely used AI applications are given below.

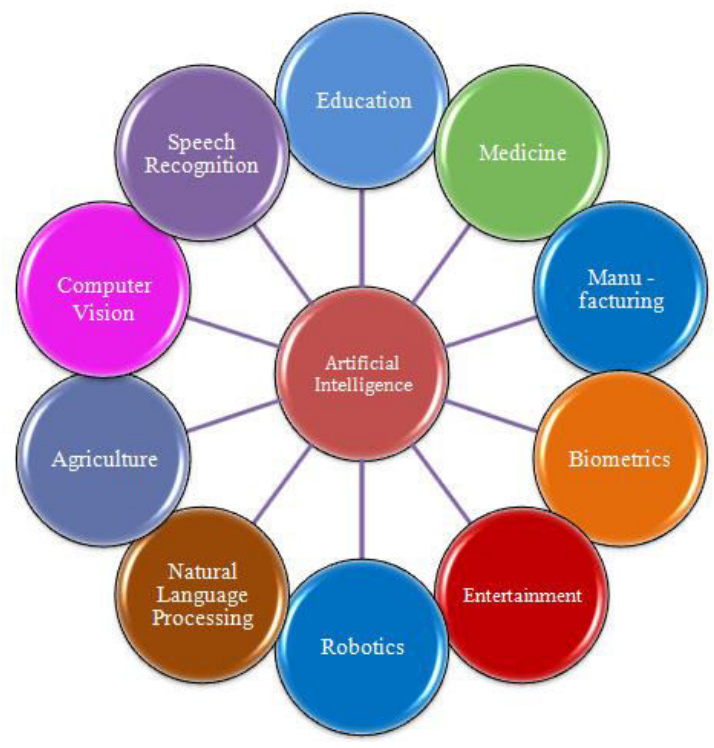

Fig. 2: Applications of Artificial Intelligence

\subsection{Medicine}

Nowadays the usage of Artificial intelligence is unavoidable in the field of medicine. AI is mostly used in most of the medical fields like Cardiology, neurology, embryology, etc. In recent years, ML algorithms are used to identify various diseases like cancer, brain tumor, malaria and dengue in earlier stage and also monitor the patient's health condition. For the last one year we are in COVID-19 Pandemic situations many researches are done to develop the drugs for Corona virus.

Computer vision is very valuable in the field of medicine. With the help of computer vision and signal processing technologies doctors can treat the patients remotely. Nowadays in most critical situation robots are used for treating the patient. It carries out hundreds of clinical tests simultaneously. It can do stitching more accurate than a human doctor. Mostly it is used for complex surgery such as brain tumors to reduce the degree of error occur [4][5].

\subsection{Agriculture}

In agriculture the advancements of AI is used to increase the crop yield also we can easily predicts the time it takes for harvest the crop thus increasing efficiency of farming. In many countries Machine learning algorithms are used to monitor the quality of soil and crops. Computer vision algorithms are used to detect crop diseases in earlier stage so the farmers can take the appropriate decision quickly. Thus increase the production quality. Nowadays robots are used in plant crops, irrigation, weed control and insect detection. Green house automation, simulation and optimization techniques are more specializations of $\mathrm{AI}$ in agriculture [6].

\subsection{Education}

Computers revolutionized the teaching field in many ways. Teachers make their notes presentation, educational quiz and videos easier. It helps the students to understand their subjects easily and more effectively. Intelligent tutoring systems increase the motivation and learning capabilities of student. Teachers use these smart and intelligent machines to prepare study materials, calculate grades, maintain attendance, access student data and evaluate student performance in online programs and assessments. Nowadays computers and internet are used for students and researchers to improve their research and communication skills. It is no doubt each and every people use search engine to clear their doubts easily. During this COVID-19 pandemic period smart phones and computers with internet are works as a major tool for e-learning.

\subsection{Natural Language Processing}

Natural language processing is not a new one, in last few years it plays a crucial role in human and machine interactions. In addition, using NLP we can do translation, information retrieval, sentiment analysis, summarization, segmentation etc. NLP techniques depend on machine learning to understand human languages [7].

\subsection{Speech Recognition}

Speech recognition is used to recognize phrases and words in our spoken language and translate it into a machine understandable format. Nowadays it is used to compose a text message and convert the text message to speech. In today's technology-driven world various DL algorithms are used in Speech recognition. 


\subsection{Robotics}

Robotics is one of the hottest sub fields of AI that is used to design intelligent machines. Robotics is mostly used in military, medicine, industries, exploration etc. In the digital age robots are a boon for the people. Due to less blood loss, less pain and scarring, less recovery time and smaller risk of infection Robots are helps to the surgeons in complex surgery.

Recently various types of robots are used in various purpose such as Industrial robots, Humanoid robots, Agriculture robots, Domestic robots, remotely controlled robots and many more. Robots are used in hazardous works like inspection of radioactive materials, bomb removal, space exploration and ocean exploration. It is very helpful in industries for handling material, cutting, welding, drilling, color coating, polishing, etc [8].

\subsection{Computer Vision}

The objective of Computer vision is to recognize the digital images. It is mostly used in medical imaging, biometrics, surveillance and object recognition. In military to detect the enemy soldiers and send the missile to the specific target area this technology is used. Computer vision is used in medicine to measure the organ dimensions, blood flow and find the structure of brain.

In addition Computer Vision and ML also used to identify the tumor in brain or other parts of the body in earlier stage [5].

\subsection{Biometrics}

Nowadays Artificial intelligence with biometrics technology is used by companies and governments in a variety of applications for identification. Emerging biometrics based identification is used in most of the security applications such as Banking security, ATM security, E-commerce etc.

\subsection{Entertainment}

Nowadays Artificial intelligence grabs user's attention in Entertainment. Using the advancement of AI technology we can play games, watch movies and sports with real effects. Today the Virtual reality technology and deep dreaming gives more interesting and immersive $3 \mathrm{D}$ visual experiences to the users in movies and games [9].

\subsection{Manufacturing}

AI plays an important role in Manufacturing. We know that drones and industrial robots have been playing a great role in manufacturing industry. AI with deep learning helps in manufacturing to lower the operational costs, increase the productivity and reduce the wastage of material. Machine learning algorithms and Complex AI algorithms for artificial neural networks are generating trustworthy predictions regarding the status of assets and machinery [10].

\section{Conclusion}

In the modern world AI is a great complement for the people. We can't imagine that a world without AI technology. AI makes our life smart. It has given precious lifestyle for us. It is the reason behind the emerging development of AI. Nowadays there are no things without AI technology. In each and every place we can see the applications of artificial intelligence such as smart television, smart refrigerator, traffic light, self driving cars etc. Nowadays most of the research is going on AI technology. In few years it will reach a great status. It is for sure that Artificial Intelligence technology will rule the world in future.

\section{References}

[1] JahanzaibShabbir, TariqueAnwer, "Artificial Intelligence and its Role in Near Future", Journal of Latex Class Files, vol.14, no.8, 2015.

[2] Pamina.J, BeschiRaja.J, "Survey On Deep LearningAlgorithms", International Journal of Emerging Technology and Innovative Engineering, vol.5, no.1, pp.38-43, 2019.

[3] Nadimpalli,M, "Artificial Intelligence Risks and Benefits" International Journal of Innovative Research in Science,Engineering and Technology, vol.6, no.6, June 2017.

[4] Pingale,K., Surwase,S., Kulkarni,V., Sarage, S., \&Karve,A.,'Disease Prediction using Machine Learning”, 2019.

[5] Dinesh Yadav, RavinSehrawat, "Artificial intelligence integration in healthcare and Medicine", International Journal Of R\&D In Engineering, Science And Management, vol.7, no.4, pp.11-17, May 2018.

[6] DikshaManaware, "Artificial Intelligence: A New way to improve Indian Agriculture", International Journal of Current Microbiology and Applied Sciences, vol.9, no.3, pp.1095-1102, 2020.

[7] Prakash M Nadkarni, LucilaOhno-Machado, Wendy W Chapman, "Natural language processing: An introduction", Journal of the American Medical Informatics Association, vol.18, no.5, pp. 544551, September 2011.

[8] Ingrand, Felix, Ghallab,Malik, "Robotics and Artificial Intelligence: a Perspective on deliberation functions", AI communications, vol.27,no.1, pp.63-80,2014.

[9] SumitDas, AritraDey, AkashPal, NabamitaRoy, "Applications of Artificial Intelligence in Machine Learning: Review and Prospect”, International Journal of Computer Applications, vol.115, no.9, April 2015 .

[10] Jinjiang Wang, Yulin Ma, Laibin Zhang, Robert X.Gao, Dazhong Wu, "Deep Learning for Smart Manufacturing: Methods and Applications", Journal of Manufacturing Systems, pp.144-156, 2018 